\title{
Towards Realistic Implementations of Large Imaging Calorimeters
}

\author{
Kiyotomo Kawagoe ${ }^{* \dagger}$ \\ Kyushu University - Department of Physics and RCAPP \\ E-mail: kawagoe@phys.kyushu-u.ac.jp \\ Lorenz Emberger ${ }^{\ddagger}$ \\ Max-Planck-Institute for Physics \\ E-mail: emberger@mpp.mpg • de
}

\begin{abstract}
The next generation of collider detectors will most likely make full use of Particle Flow algorithms, requiring precision tracking and imaging calorimeters. The latter, with granularity 2 to 3 orders of magnitude above existing devices, have been developed during the last 15 years by the CALICE collaboration and are now approaching maturity. The state-of-the-art and the remaining challenges will be presented for all the investigated readouts: silicon diodes and scintillator for a tungsten electromagnetic calorimeter, gaseous with semi-digital readout and scintillator with SiPM readout for a hadronic one. We will describe the commissioning, including beam tests, of large scale technological prototypes of a silicon tungsten electromagnetic calorimeter and hadron calorimeters featuring either a gaseous medium or scintillator with SiPM as an active material. Where applicable, raw performances of calorimeter such as energy resolution and linearity will be presented but also studies exploiting the distinct features of granular calorimeters regarding pattern recognition. Beyond these prototypes, the design of experiments addressing the requirements and potential of imaging calorimetry will be commented on.
\end{abstract}

XXIX International Symposium on Lepton Photon Interactions at High Energies - LeptonPhoton2019 August 5-10, 2019

Toronto, Canada

\footnotetext{
${ }^{*}$ Presenter.

$\dagger$ On behalf of the CALICE collaboration

$\ddagger$ On behalf of the CALICE collaboration
} 


\section{Introduction}

To fully expolit the potential of future $\mathrm{e}^{+} \mathrm{e}^{-}$collider experiments, a precise reconstruction of all final states is necessary. To achieve this for hadronic final states, a jet energy resolution significantly beyond the current state-of-the-art, in the order of 3\% - 4\% over a wide energy range, is required. In contrast to traditional jet energy measurement of summing up the energy depositions in all sub-detectors, the Particle Flow (PF) approach is designed to measure different types of particles of a jet in the best suited detector. The tracker for charged particles, the electromagentic calorimeter (ECAL) for photons and the hadronic one (HCAL) for neutral hadrons. Hence, only $10 \%$ jet energy carried by neutral hadrons is measured in the HCAL, typically offering a worse energy resolution. To reduce double counting of charged particles in the tracker and calorimeter, the spatial resolution in the calorimeters has to be high enough to seperate particle showers [7]. The CALICE collaboration is focussed on the design and realization of such calorimeters.

\section{Prototypes of the CALICE Calorimeters}

The concepts considered in this contribution are two analog ECAL and two HCAL prototypes. These prototypes are called technological prototypes because they address constraints of realistic detector scenarios like compactness, power consumption and structural integrity over large volumes. The technological prototypes feature power pulsing capability switching the electronics on and off in syncronization with the collision of a bunch train in a linear collider to reduce power consumption. A common family of read out ASICs (HARDROC, SPIROC, SKIROC) [3] is used in CALICE to facilitate combined running and data acquisition of the different prototypes.

\subsection{Electromagnetic Calorimeters}

A scintillator ECAL (Sc-ECAL) [4], which is currently under development, features crossed scintillator strips read out by silicon photomultipliers ( $\mathrm{SiPM}$ ) interleaved with metal absorbers. The effective granularity will be $5 \times 5 \mathrm{~mm}^{2}$.

The silicon tungsten ECAL (SiW-ECAL) [2] prototype consists of 15 subsequent layers of tungsten absorber and $9 \times 9 \mathrm{~cm}^{2}$ silicon wafers each hosting $5.5 \times 5.5 \mathrm{~mm}^{2}$ pixels. Despite the limited number of layers, this prototype successfully demonstrates the technological developments towards an integration in a full collider detector.

\subsection{Hadronic Calorimeters}

The analog hadronic calorimeter (AHCAL) [6] consists of 39 successive layers of a $17 \mathrm{~mm}$ thick steel absorber and a $3 \mathrm{~mm}$ thick scintillator as active material. The active material is segmented in $3 \times 3 \mathrm{~cm}^{2}$ tiles to achieve high granularity. This "SiPM-on-tile" technology features tiles which are individually wrapped in reflective foil and mounted directly on a printed circuit board hosting a SiPM.

The gaseous semi-digital hadronic calorimeter (SDHCAL) [1] features 48 layers of steel absorbers interleaved with $1 \mathrm{~m}^{2}$ Glass Resistive Plate Chambers (GRPC) as active medium. The GRPCs charge is collected by $1 \mathrm{~cm}^{2}$ copper electrodes and the read out is operated in 2 bit mode resulting in 3 thresholds. 


\section{Performance in Beam Tests}

The CALICE Prototypes are extensively tested in beam test facilities recording muon, electron and hadron data. Due to the high spatial granularity it is possible to record images of electromagnetic and hadronic showers as shown in fig. 1 on the left. This feature enhances the capability of the calorimeters to separate close by showers, an essential feature of PF detectors. The single cell timing capability of the prototypes is a key feature of future collider detectors where high luminosity and beam-induced backgrounds cause increased pile up of background events. Another essential requirement for $\mathrm{PF}$ is the ability to trigger on and read out small energy depositions demanding a sufficient signal to noise $(\mathrm{S} / \mathrm{N})$ ratio. The right part of fig. 1 shows a noise peak around 3 ADC counts alongside a MIP measurement around 60 ADC in the SiW-ECAL. The resulting S/N of 20 [2] is enough to discriminate energy depositions at the MIP level and implies a sufficient $\mathrm{S} / \mathrm{N}$ also in the trigger channels.
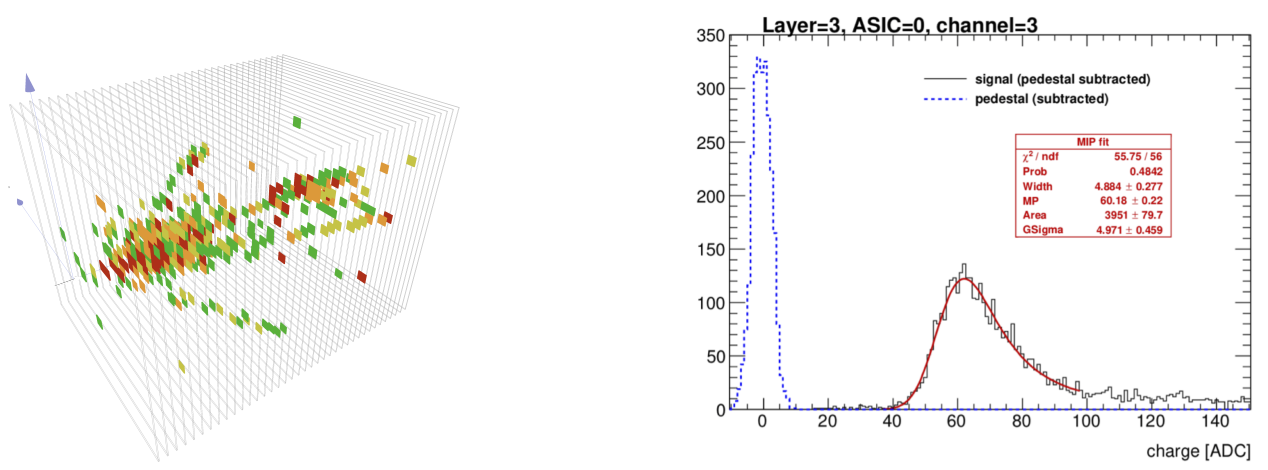

Figure 1: On the left, an image of a $60 \mathrm{GeV}$ pion hadronic shower in the AHCAL is shown. Red indicates high energy deposition, green indicates low energy deposition. On the right, a distribution of noise signals around 3 ADC counts is shown alongside the distribution of MIP energy depositions in the SiW-ECAL [2]. This results in a signal to noise ratio of 20.

\section{Recent Mechanical and Electronic Developments}

The main purpose of the technological prototypes is the development and testing of methods to integrate the detectors in large collider detector systems, which require to meet spatial constraints as well as power and cooling limits while keeping the performance. The scalability of the comparably small prototypes of about $1 \mathrm{~m}^{2}$ front face area to complete barrel and end cap modules has to be established. As an example, the RPC plates of the SDHCAL rely on a homogeneous gas flow throughout the whole volume. A sophisticated gas circulation system is shown on the left side of fig. 2 providing a homogeneous gas distribution and volume exchange over a $2 \mathrm{~m}^{2}$ area. To distribute the power for the detector over these large areas, a powerful but compact electronic system is needed. The right side of fig. 2 shows the detector interface (DIF) and power supply boards at the end of a more than $2 \mathrm{~m}$ long AHCAL module. The stable behaviour of the set up allows the assembly of full size detectors. Similar developments also exist for the SiW-ECAL technology. 

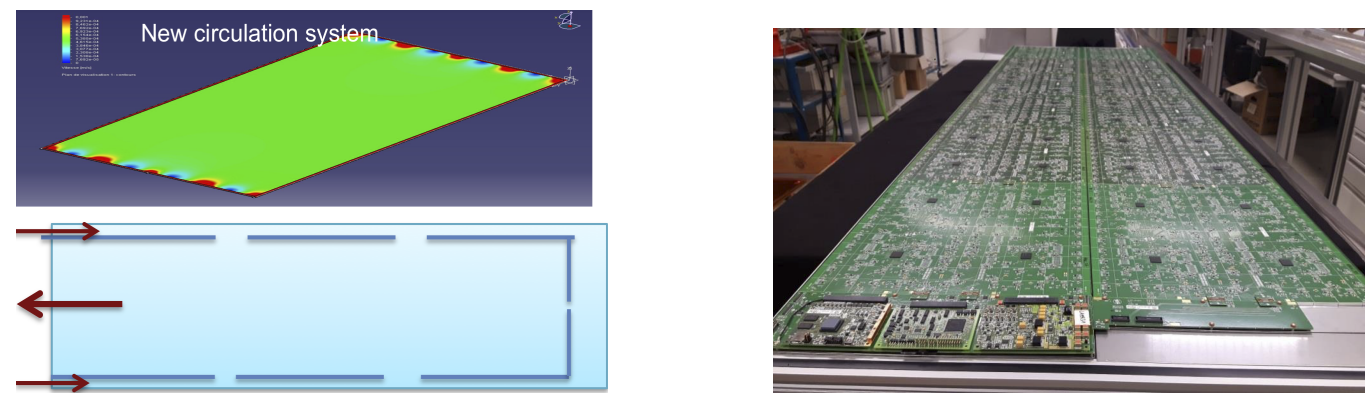

Figure 2: Left: Improved gas distribution scheme in the RPC of the SDHCAL ensuring a homogeneous velocity profile of the gas flow. Right: Detector interface (DIF) and power supply boards of the AHCAL powering and reading a more than 2 meter long detector module containing 1728 channels.

\section{Conclusion}

The calorimeters developed by CALICE are optimized for Particle Flow event reconstruction for future $\mathrm{e}^{+} \mathrm{e}^{-}$colliders. Extensive beam tests of the various prototypes validated the compact design features and capabilities of the calorimeters. The expertise of CALICE is already applied in detector upgrades of the upcoming HL-LHC e.g. the CMS HGCAL [5].

\section{References}

[1] Guillaume Baulieu et al. "Construction and commissioning of a technological prototype of a high-granularity semi-digital hadronic calorimeter". In: Journal of Instrumentation 10 (June 2015). DOI: $10.1088 / 1748-0221 / 10 / 10 /$ P10039.

[2] S. Bilokin et al. "Commissioning of the highly granular SiW-ECAL technological prototype". In: (2018). arXiv: 1810.05133 [physics.ins-det] .

[3] S. Callier et al. "ROC chips for imaging calorimetry at the International Linear Collider". In: JINST 9 (2014), p. C02022. DOI: $10.1088 / 1748-0221 / 9 / 02 / C 02022$.

[4] K. Francis et al. "Performance of the first prototype of the CALICE scintillator strip electromagnetic calorimeter". In: Nucl. Instrum. Methods Phys. Res., A 763.arXiv:1311.3761 (Nov. 2013), 278-289. 12 p. DOI: $10.1016 /$ j . nima. 2014.06 .039 . URL: http: //cds.cern.ch/record/1629400.

[5] Christophe Ochando. "HGCAL: A High-Granularity Calorimeter for the endcaps of CMS at HL-LHC". In: J. Phys. : Conf. Ser. 928.1 (2017), 012025. 4 p. DOI: 10 . $1088 / 1742-$ 6596/928/1/012025. URL: https://cds.cern.ch/record/2311394.

[6] Felix Sefkow and Frank Simon. "A highly granular SiPM-on-tile calorimeter prototype”. In: Journal of Physics: Conference Series 1162 (Jan. 2019), p. 012012. DOI: 10 . $1088 / 1742-$ 6596/1162/1/012012. URL: http://dx.doi.org/10.1088/1742-6596/ $1162 / 1 / 012012$.

[7] Felix Sefkow et al. "Experimental Tests of Particle Flow Calorimetry". In: Rev. Mod. Phys. 88 (2016), p. 015003. DOI: 10.1103 /RevModPhys. 88.015003 . arXiv: 1507.05893 [physics.ins-det]. 